\title{
A Brain Emotional Learning-based Prediction Model for the Prediction of Geomagnetic Storms
}

\author{
Mahboobeh Parsapoor ${ }^{1,2}$, Urban Bilstrup ${ }^{1}$, Bertil Svensson ${ }^{1}$ \\ ${ }^{1}$ School of Information Science, Computer and Electrical \\ Engineering (IDE),Halmstad University, Halmstad, Sweden \\ ${ }^{2}$ School of Computer Science, Faculty of Engineering \& Physical \\ Science, The University of Manchester, Manchester, UK \\ ${ }^{1}$ \{Mahboobeh.Parsapoor, Urban.Bilstrup, Bertil.Svensson \}@hh.se \\ ${ }^{2}$ mahboobeh.parsapoor@postgrad.manchester.ac.uk
}

\begin{abstract}
This paper introduces a new type of brain emotional learning inspired models (BELIMs). The suggested model is utilized as a suitable model for predicting geomagnetic storms. The model is known as BELPM which is an acronym for Brain Emotional Learning-based Prediction Model. The structure of the suggested model consists of four main parts and mimics the corresponding regions of the neural structure underlying fear conditioning. The functions of these parts are implemented by assigning adaptive networks to the different parts. The learning algorithm of BELPM is based on the steepest descent (SD) and the least square estimator (LSE). In this paper, BELPM is employed to predict geomagnetic storms using the Disturbance Storm Time (Dst) index. To evaluate the performance of BELPM, the obtained results have been compared with the results of the adaptive neuro-fuzzy inference system (ANFIS).
\end{abstract}

\section{INTRODUCTION}

$\mathrm{T}$ HE geomagnetic storm, which originates from the solar wind, disturbs the Earth's magnetosphere and has caused harmful damage to the ground based communication, electricity power network, etc. Therefore, developing alert systems for geomagnetic storms is essential in order to prevent these harmful effects [1]-[6].

The disturbance storm time, Dst, is one of the main indices of a geomagnetic storm and was defined by Bruce Tsurutani [1], [5]-[6]. It is a measurement to count 'the number of solar charged particles that enter the Earth's magnetic field' [6]. The Dst index has been proposed to characterize the phases of geomagnetic storms i,e., the initial phase, main phase and recovery phase and has been recorded by several space centers such as the World Data Center for Geomagnetism, Kyoto, Japan.

Different machine learning methods e.g., linear input-output techniques or linear prediction filtering neural networks [8][9], neurofuzzy methods [2],[4], have been investigated for predicting geomagnetic storms using the Dst index [1][4], [6]-[12]. Amongst them, neural networks and neuro-fuzzy models have shown high generalization [13],[14] capabilities to model nonlinear behavior of the Dst index .

Recently, inspiration from the mammalian emotional systems to develop emotion-based models has received fairly good attention [15]-[25]. The emotion-based models that were proposed in [15]-[17] have been developed by a limited modification of a computational model of emotional learning that is referred to as 'amygdala-orbitofrontal system'; this computational model simulates the emotional learning in the amygdala (i.e.,one region of the mammalian brain) [26]. The obtained results from [15]-[17] verify that there are not able to accurately predict chaotic behavior of nonlinear systems.

This paper suggests a new instance of Brain Emotional Learning-Inspired Models (BELIMs) that are emotion-based models. The suggested model is applied to predict the Dst index of geomagnetic storms. So far different variations of BELIMs have been [18]-[25] examined for forecasting solar activity and geomagnetic storms.

The main contribution of this paper is to present a new version of BELIMs to be used as an accurate prediction method for the long horizon prediction of the Dst index. Another contribution of this paper is to provide comparative results when predicting the Dst index.

The rest of the paper is organized as follows: Section II reviews related works to emotions. Section III describes the structure, function and learning algorithm of the BELPM (Brain Emotional Learning Based Prediction Model). Section IV reviews the related studies in predicting the Dst index and the results of BELPM to predict the Dst index are described. Finally, conclusions about the performance of BELPM and the further improvements to the suggested model are discussed in Section V.

\section{Related Works To 'EMOtions'}

Neuroscientists and psychologists have tried to describe emotions on the basis of different hypotheses, e.g., psychological, neurobiological, philosophy, and learning hypotheses [27]. Cognitive neuroscientists have also tried to describe the neural system underlying the emotional process. One of the earlier works is the 'Papez circuit' (See Fig. 1) that was proposed by Jamez Papez. As Fig. 1 shows, this circuit includes the 'hypothalamus, anterior thalamus, cingulate gyrus and hippocampus' [27]. 


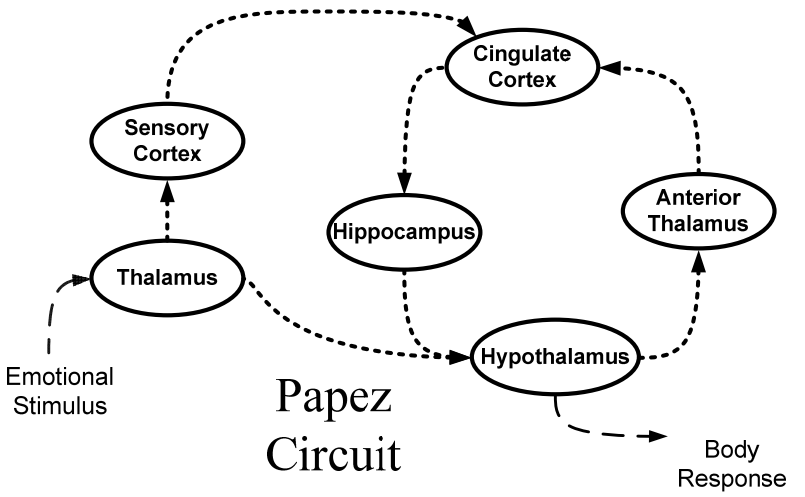

Fig.1. The Papez Circuit and its component.

MacLean modified the Papez circuit and proposed the limbic system theory to describe the regions of the brain that are responsible for emotional processing. The limbic system includes the hippocampus, amygdala, thalamus and sensory cortex [27]. Later, neuroscientists rejected the limbic system theory and stated that different parts of the brain are responsible for different emotional behavior [28]. Fear is a common emotional behavior that exists as well for humans as animals. Fear conditioning has been defined as a 'behavioral paradigm' which means learning fearful stimuli to predict aversive events [28].

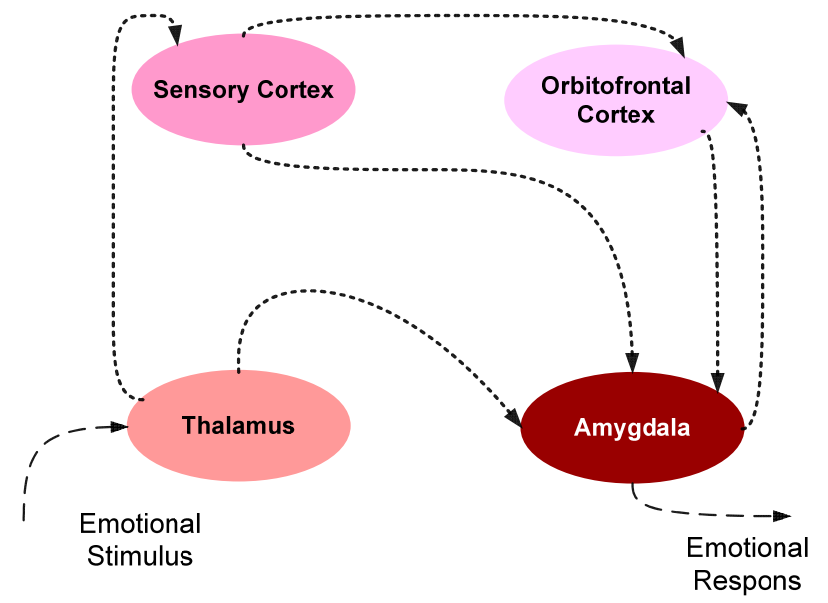

Fig. 2. A schematic of the brain's parts and their interconnections in fear conditioning.

Figure 2 displays how the amygdala and other parts of the brain, thalamus, sensory cortex and orbitofrontal cortex connected to process a fearful stimulus in the mammalian brain. As the diagram indicates, the amygdala is the central part to process the emotional stimulus. The neural structures of emotional behavior have been the foundation of the computational model of emotional learning.

Computational models of emotion [23],[25],[26] are computer-based models that have been developed to simulate different aspects of the emotional system. A good example of computational models is a model that is referred to as amygdala-orbitofrontal system and has been proposed to simulate emotional learning in the amygdala [26].

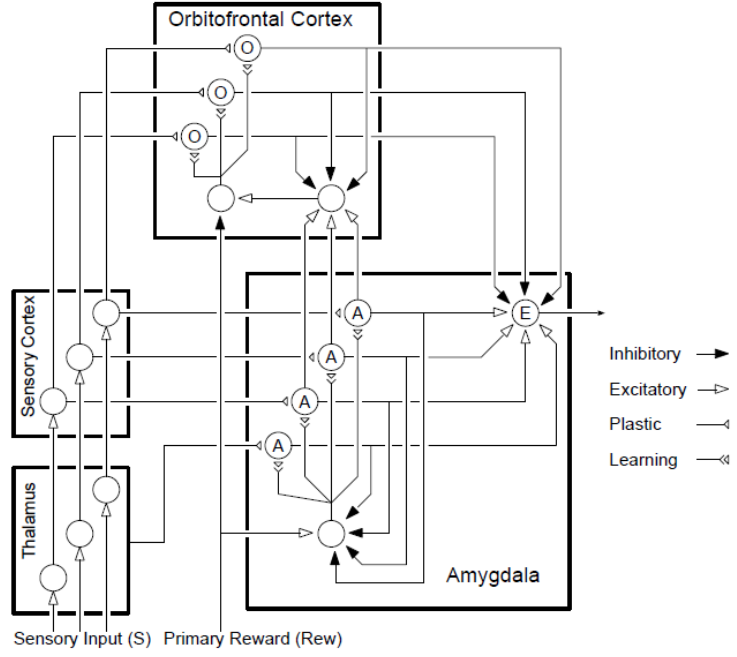

Fig.3. The Amygdala-orbitofrontal system [26].

Figure 3 depicts the internal structure of the amygdala-orbitofrontal system and describes that orbitofrontal cortex and amygdala consists of several nodes; the output of each node of the amygdala is represented as $A_{i}$ while the output of each node of the orbitofrontal cortex is represented as $\mathrm{O}_{i}$. The overall output of the model is represented as $\mathbf{E}$ and it is formulated as equation (1) [26].

$\mathbf{E}=\sum \mathrm{A}_{\mathrm{i}}-\sum \mathrm{O}_{\mathrm{i}}$

Here $\mathrm{A}_{i}$ and $\mathrm{O}_{i}$ are the output of the $i^{\text {th }}$ node of the amygdala and the orbitofrontal cortex, respectively. The updating rules of the model are based on the reinforcement signal REW . The updating rules are formalized as equations (2) and (3) and are utilized to adjust the weights $\mathbf{V}$ and $\mathbf{W}$ that are associated with the nodes of the amygdala and the orbitofrontal part, respectively [26]. Here $s_{i}$ is the input stimulus for the $i^{\text {th }}$ node of the amygdala and the orbitofrontal cortex [26].

$\Delta \mathrm{V}_{\mathrm{i}}=\alpha\left(\mathrm{S}_{\mathrm{i}} \times \max \left(0, \mathbf{R E W}-\sum \mathrm{A}_{\mathrm{i}}\right)\right)$

$\Delta \mathrm{W}_{\mathrm{i}}=\beta\left(\mathrm{S}_{\mathrm{i}} \times\left(\sum \mathrm{O}_{\mathrm{i}}-\mathbf{R E W}\right)\right)$

The amygdala-orbitofrontal model [26] has a simple structure and has been used as a foundation for numerous 1 emotion-based models [15]-[25],[29]-[33]. As was discussed earlier, the emotion-based models in [15]-[16] were proposed as chaotic time series prediction models. The foundation of these models is amygdala-orbitofrontal system; however they were developed by changing the updating rules of amygdala-orbitofrontal system. These models have not shown good results to accurately predict chaotic time series [15]-[16]. In [17], another modification of amygdala-orbitofrontal system was proposed by changing the input vector of the thalamus and the amygdala part; in addition, the updating rules of amygdala and orbitofrontal cortex were modified. The model that is named 'ADBEL' [17] 
(adaptive brain emotional decayed learning) was applied to predict the hourly Dst index; however, the obtained results verify the ADBEL could not accurately predict chaotic behavior of the Dst index.

The Emotion-based controllers [29]-[33] have also been developed by imitating the structure of the amygdala-orbitofrontal.

This paper presents a new type of emotion-based prediction models. Although the general structure of this model is similar to the amygdala-orbitofrontal system, the internal structure of this model is different from the amygdala-orbitofrontal system.

\section{BRAIN EMOTIONAL LEARNING-BASED PREDICTION MODEL}

The Brain Emotional Learning-Based Prediction Model (BELPM) is a type of Brain Emotional Learning-Inspired Model (BELIM) which is a new category of computational intelligence models. The general structure of a BELIM is an extension of the amygdala-orbitofrontal system by adding new internal parts that have been inspired by the neural structure of one of the emotional systems in the brain. Different types of BELIM: Brain Emotional Learning-based Fuzzy Inference System (BELFIS), Brain Emotional Learning-based Recurrent Fuzzy System (BELRFS) and Emotional Learning Inspired Ensample Classifier (ELiEC) [18]-[25] have been proposed as prediction models and classification models.

\section{A. Structural Aspect of BELPM}

Figure 4 depicts the structure of BELPM and shows that it consists of four main parts: TH, CX, AMYG and ORBI which refer to the THalamous, sensory CorteX, AMYGdala, and ORBItofrontal cortex, respectively. The structure of BELPM copies the interconnection of those parts (THalamous, sensory CorteX, AMYGdala, and ORBItofrontal cortex) that are responsible to process the emotional stimuli. It should be noted that these regions of the brain are very complex and there is no intention to mimic their functionality and all their connections in detail.

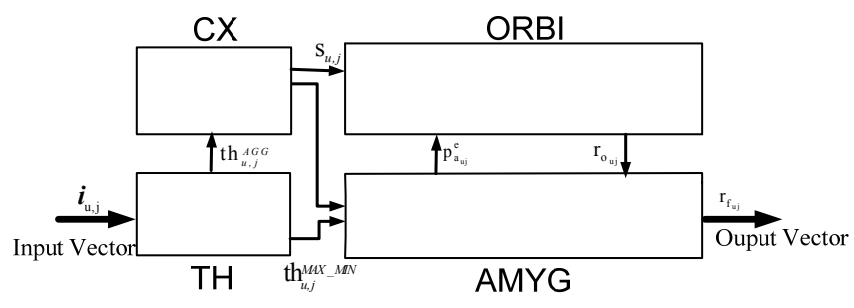

Fig. 4. The structure of BELPM.

The following steps describes the input and output of each part of BELPM; when it receives an input as $i_{u, j}$ from the training data set, $\mathbf{I}_{\mathrm{u}}=\left\{\mathbf{i}_{\mathrm{u}, \mathrm{j}}\right\}_{\mathrm{j}=1}^{\mathrm{N}_{\mathrm{u}}}$.
1. First, $\mathbf{i}_{\mathrm{u}, \mathrm{j}}$ the $\mathrm{j}^{\text {th }}$ input vector from $\mathbf{I}_{\mathrm{u}}=\left\{\mathbf{i}_{\mathrm{u}, \mathrm{j}}\right\}_{\mathrm{j}=1}^{\mathrm{N}_{\mathrm{u}}}$ (taking the assumption that the number of training samples is equal to $\mathrm{N}_{\mathrm{u}}$; the subscript $\mathrm{u}$ has been used to determine the input data is chosen from the training data set) enters the $\mathrm{TH}$, which provides two outputs, $\mathbf{t h}_{\mathrm{u}, \mathrm{j}}^{\mathrm{Max}}{ }^{\mathrm{Min}}$ and $\mathbf{t h}_{\mathrm{u}, \mathrm{j}}^{\mathrm{AGG}}$ which are sent to the AMYG and the CX, respectively.

2. The CX provides $\mathbf{s}_{\mathrm{u}, \mathrm{j}}$ and sends it to both the AMYG and the ORBI.

3. The AMYG receives two inputs: $\mathbf{t h}_{\mathrm{u}, \mathrm{j}}^{\mathrm{Max}}{ }^{\mathrm{Min}}$ and $\mathbf{s}_{\mathrm{u}, \mathrm{j}}$. It provides the primary output, $\mathrm{r}_{\mathrm{a} u \mathrm{j}}$, and expected punishment, $\mathrm{p}_{\mathrm{a} u, j}^{\mathrm{e}}$, that is sent to the ORBI (the subscript a has been used to show the outputs of AMYG).

4. The ORBI receives $\mathbf{s}_{\mathrm{u}, \mathrm{j}}$ and $\mathrm{p}_{\mathrm{a}, \mathrm{j}}^{\mathrm{e}}$. It provides the secondary output, $\mathrm{r}_{\mathrm{o}_{\mathrm{u}, \mathrm{j}}}$, and sends it to the AMYG. 5. The AMYG receives $r_{o_{u, j}}$ and provides the final output, $r_{f u, j}$ (the subscript $\mathrm{f}$ has been used to show the final outputs).

\section{B. Functional Aspect of BELPM}

The function of BELPM is implemented by assigning adaptive networks to different parts. Figure 5 describes how the adaptive networks can be assigned to each part to implement the functionality of that part.

The adaptive network (see Fig. 6) consists of a number of nodes that are connected by directional links. The nodes of the adaptive network can be classified into circle and square nodes. A circle node has a function without adjustable parameters; in contrast, the square nodes have been defined by a function with the adjustable parameters. The learning parameters of an adaptive network are a combination of linear and nonlinear parameters and can be adjusted by using a learning algorithm.

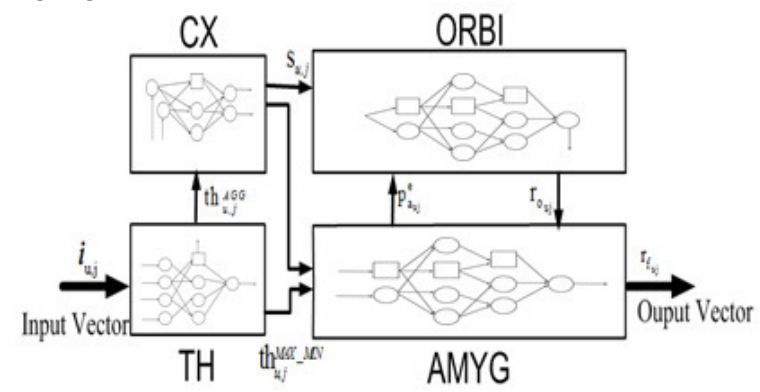

Fig. 5. Assigning different adaptive networks to different parts of BELPM. 


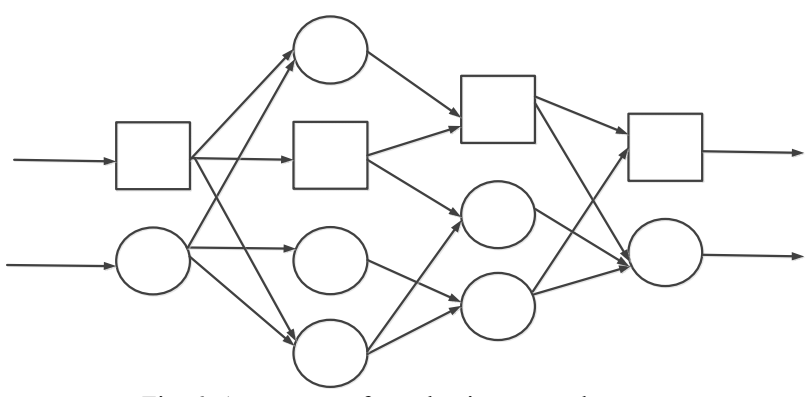

Fig. 6. A structure of an adaptive network.

C. A Weighted k-Nearest Neighbor based Adaptive Network

In BELPM, weighted k-nearest neighbor based adaptive networks are assigned to the AMYG and the ORBI. This adaptive network has been developed on the basis of weighted k-Nearest Neighbor (Wk-NN). Figure 7 describes a simple Wk-NN based adaptive network and shows that it is divided into four layers. In the following, the function, the input and the output of each layer has been explained.

The first layer consists of $\mathrm{k}$ square nodes with $\mathrm{K}($.) function (kernel function). Note that in Fig. $7, \mathrm{k}$ is equal to three. This layer has an input vector as $\mathbf{d}_{\min }=\left\{\mathrm{d}_{\mathrm{min}, \mathrm{m}}\right\}_{\mathrm{m}=1}^{\mathrm{k}}$. The $\mathbf{d}_{\min }$ is a set of $\mathrm{k}$ minimum distances of $\mathbf{d}=\left\{\mathrm{d}_{\mathrm{j}}\right\}_{\mathrm{j}=1}^{\mathrm{N}_{\mathrm{u}}}$. The distances vector $\mathbf{d}=\left\{\mathrm{d}_{\mathrm{j}}\right\}_{\mathrm{j}=1}^{\mathrm{N}_{\mathrm{u}}}$ can be calculated as Euclidean distances between a new input as $\mathbf{i}_{c, j}$ and the training data as $\left\{\mathbf{i}_{\mathrm{u}, \mathrm{j}}\right\}_{\mathrm{j}=1}^{\mathrm{N}_{\mathrm{u}}}$. The output vector of the $\mathrm{m}^{\text {th }}$ node of the first layer is calculated using equation (4). Here, the input to the $\mathrm{m}^{\text {th }}$ node is $\mathrm{d}_{\text {min, } \mathrm{m}}$.

$\mathrm{n}_{\mathrm{m}}^{1}=\mathrm{K}\left(\mathrm{d}_{\mathrm{min}, \mathrm{m}}\right)$

In general, the kernel function for the $\mathrm{m}^{\text {th }}$ node can be one of the functions that have been defined as equations (5), (6), and (7). The input and the parameter of $\mathrm{K}($.$) of \mathrm{m}^{\text {th }}$ node can be determined using $d_{m}$ and $b_{m}$.

$\mathrm{K}\left(\mathrm{d}_{\mathrm{m}}\right)=\exp \left(-\mathrm{d}_{\mathrm{m}} \mathrm{b}_{\mathrm{m}}\right)$

$\mathrm{K}\left(\mathrm{d}_{\mathrm{m}}\right)=\frac{1}{\left(1+\left(\mathrm{d}_{\mathrm{m}} \mathrm{b}_{\mathrm{m}}\right)^{2}\right)}$

$\mathrm{K}\left(\mathrm{d}_{\mathrm{m}}\right)=\frac{\max (\mathbf{d})-\left(\mathrm{d}_{\mathrm{m}}-\min (\mathbf{d})\right)}{\max (\mathbf{d})}$

The second layer is a normalization layer and has $\mathrm{k}$ nodes (fixed or circle), which are labeled $\mathrm{N}$ to calculate the normalized values of $\mathbf{n}^{1}$ using (8). The input vector of this layer is $\mathbf{n}^{1}$ and the output of $\mathrm{m}^{\text {th }}$ node in this layer can be calculated as (8)

$\overline{\mathrm{n}}_{\mathrm{m}}^{1}=\frac{\left(\mathrm{n}_{\mathrm{m}}^{1}\right)}{\sum_{\mathrm{m}=1}^{\mathrm{k}} \mathrm{n}_{\mathrm{m}}^{1}}$

The third layer has $\mathrm{k}$ circle nodes; the function of $\mathrm{m}^{\text {th }}$ node of this layer is given in (9). This layer has two input vectors, $\overline{\mathbf{n}}^{1}$ and $\mathbf{r}_{\text {min, }}$; the latter is a vector that is extracted from $\mathbf{r}_{\mathrm{u}}=\left[\mathrm{r}_{\mathrm{u}, 1}, \mathrm{r}_{\mathrm{u}, 2}, \ldots, \mathrm{r}_{\mathrm{u}, \mathrm{N}_{\mathrm{u}}}\right]$ and is related to the target outputs of the $\mathrm{k}$ samples of $\left\{\mathbf{i}_{\mathrm{u}, \mathrm{j}}\right\}_{\mathrm{j}=1}^{\mathrm{N}_{\mathrm{u}}}$ that have minimum distances with the new input $\mathbf{i}_{c, j}$. The output of this layer is $\mathbf{n}^{3}$.

$\mathrm{n}_{\mathrm{m}}^{3}=\frac{\left(\mathrm{n}_{\mathrm{m}}^{1}\right)}{\sum_{\mathrm{m}=1}^{\mathrm{k}} \mathrm{n}_{\mathrm{m}}^{1}} \times \mathrm{r}_{\mathrm{u}, \mathrm{m}}$

The fourth layer has a single node (circle) that performs the summation of the input vector, $\mathbf{n}^{3}$ to produce $\mathbf{r}$.

The above explanation has illustrated the function of a simple Wk-NN based adaptive network. It should be noted that in BELPM, the AMYG and the ORBI are assigned this type of adaptive network.

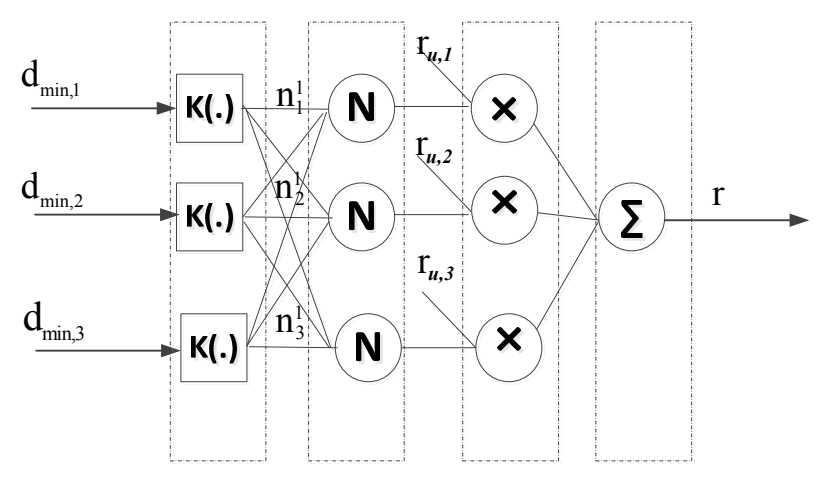

Fig.7. A weighted k-Nearest Neighbor adaptive network.

\section{Learning Aspect of BELPM}

To adjust the linear and nonlinear learning parameters, a hybrid learning algorithm that consists of the steepest descent (SD) and the least-squares estimator (LSE) is used. The SD updates the nonlinear parameters in a gradient related direction to minimize the loss functions, which are defined based on the outputs of the adaptive networks. The LSE is applied to update the linear parameters. The learning algorithm has been explained in detail in [25].

\section{Related wORK to Predict GeOMAgnetic Storms}

As was previously discussed, developing an alert system for geomagnetic storms is essential. The Dst index that has been defined to measure the intensity of geomagnetic storms has been utilized by many data driven models. The following subsection reviews several studies that have been used to predict geomagnetic storms.

\section{A. Related works to Predict Geomagnetic Storm}

A good review of earlier studies related to use the Dst index to predict geomagnetic storms have been done in [6]. The authors of [6] provided a survey of using the Dst index to predict geomagnetic storms; they also proposed a neural network-based prediction model to predict the minimum 
values of the Dst index. The model was successfully evaluated to predict geomagnetic storms of 1980 and 1989. In [9], a recurrent neural network was introduced to predict one hour step of Dst from 2001. The authors of [9] also showed that combining principal component analysis (PCA) and NN could significantly increase the accuracy of prediction of a geomagnetic storm. The damage and harmful effects of geomagnetic storms were reviewed in [10]. The authors of [10] studied the effect of the embedding dimension on the chaotic behavior of the Dst time series; the proposed model in [10] was is study tested for two super storms: 13 March 1989 and 11 January 1997. In [2] a combination of Singular Spectrum Analysis (SSA) and locally linear neuro-fuzzy model was proposed as a useful methodology to increase the accuracy of long term prediction of Dst time series. Specifically, this method was examined to predict ten steps ahead of extracted Dst time series between 1988 and 1990, Within this time window the geomagnetic storm damaged Quebec's power grid and caused a blackout in Quebec [10] is included. A nice review of the Dst index prediction models and the benefits of prediction of the Dst index was presented in [12]. The authors of [12] also proposed a long term prediction model that is known as 'Anemomils'[12] and tested it for three geomagnetic storms of 2001, 2005 and 2012.

\section{B. Evaluating the BELPM's Performance on the Dst index}

This subsection evaluates BELPM's performance by examining it on two sets of the Dst index. The code of BELPM has been written in MATLAB and the Dst index can be downloaded from the World Data Center for Geomagnetism, Kyoto, Japan.

The first data set is related to the Dst index of the super storm that occurred in March 1989. The value of the Dst index on the $13^{\text {th }}$ March of 1989 reached to ' -589 nanotesla (nT)'. Figure 8 depicts the hourly DST index during January1988 to January 1990.

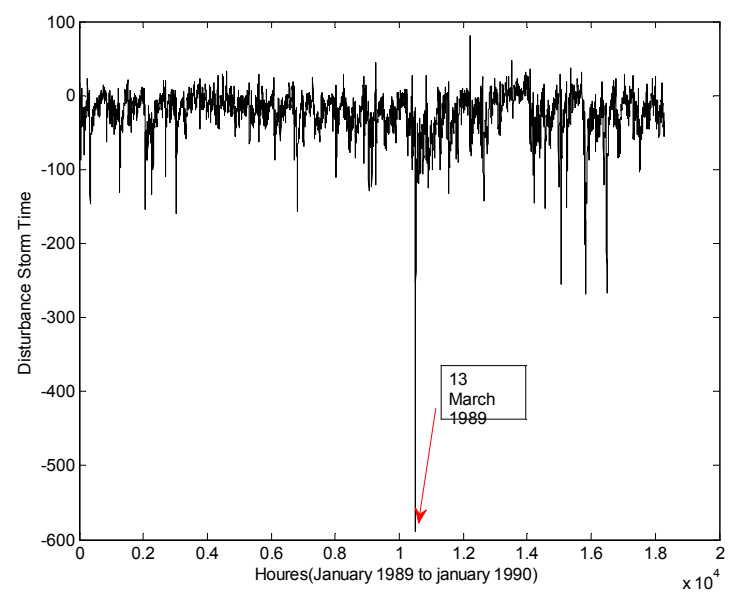

Fig. 8. The hourly Dst index from January 1988 to January 1990.

The second data set is related to the geomagnetic super storm which occurred on July $15^{\text {th }}$ to $17^{\text {th }}$; the minimum of the Dst index was - 301 nanotesla (nT). Figure 9 depicts the hourly DST index during the days of July 2000.

To provide a careful comparison with other methods, this paper utilizes two error measures: normalized mean square error (NMSE) and the correlation coefficient $\rho_{\mathrm{y}, \hat{\mathrm{y}}}$ that are given as equations (10) and (11).

NMSE $=\frac{\sum_{j=1}^{N}\left(y_{j}-\hat{y}_{j}\right)^{2}}{\sum_{j=1}^{N}\left(y_{j}-\bar{y}_{j}\right)^{2}}$

$\rho_{\mathrm{y}, \hat{\mathrm{y}}}=\frac{\operatorname{Cov}(\mathrm{y}, \hat{\mathrm{y}})}{\sigma_{\mathrm{y}} \sigma_{\hat{\mathrm{y}}}}$

The parameters $\hat{\mathbf{y}}$ and $\mathbf{y}$ refer to the predicted values and desired targets, respectively. The parameter $\overline{\mathbf{y}}$ is the average of the desired targets.

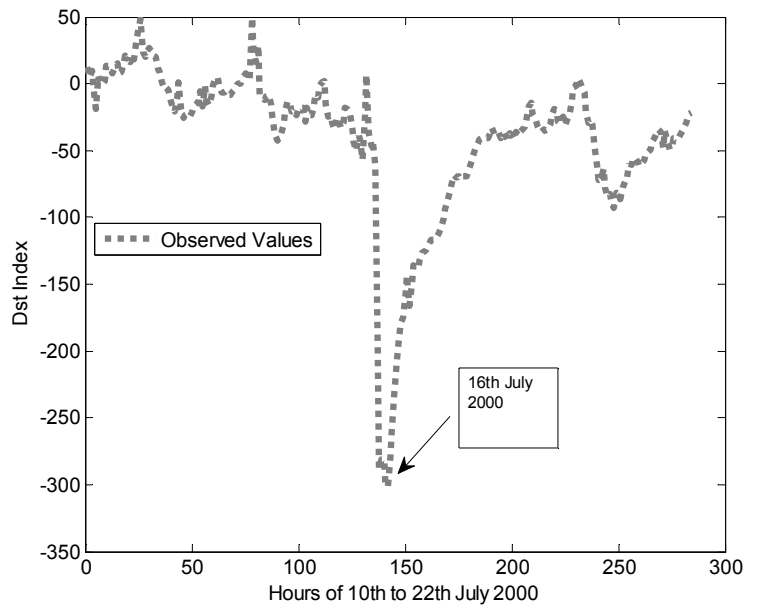

Fig.9. The hourly Dst index for July 2000.

As was mentioned earlier, in the first experiment, BELPM is tested to model the Dst time series between january1988 to January 1990. The Dst time series is related to one of the harmful geomagnetic storms which occurred during solar cycle 22 and caused severe damage to Quebec's electricity power system. In this case, the embedded dimension is selected as three. Figure 10 shows the inputs and output of BELPM as a black box to predict the Dst time series.

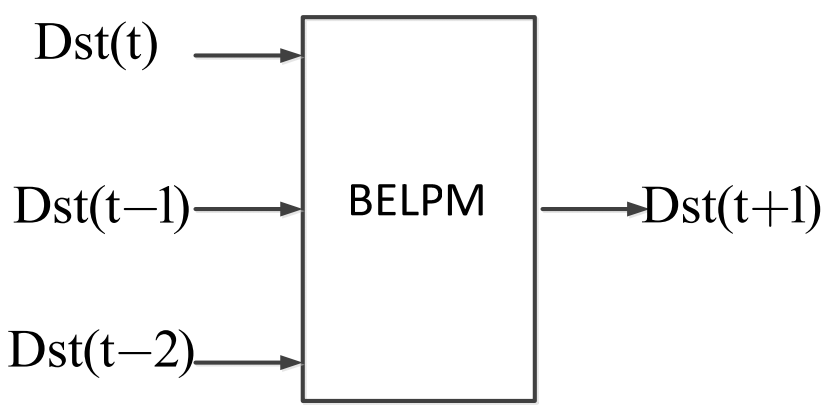

Fig.10. A black box of the BELPM to receive the Dst index and predict one step ahead of the Dst index. 
The predicted values of the Dst index versus the observed values of the first data set includes the Dst index of $13^{\text {th }}$ March 1989 which has been described in Fig.11(a). The training data samples of Dst index has been depicted in Fig. 11(b). This figure shows that BELPM can outperform ANFIS in modeling the Dst index.

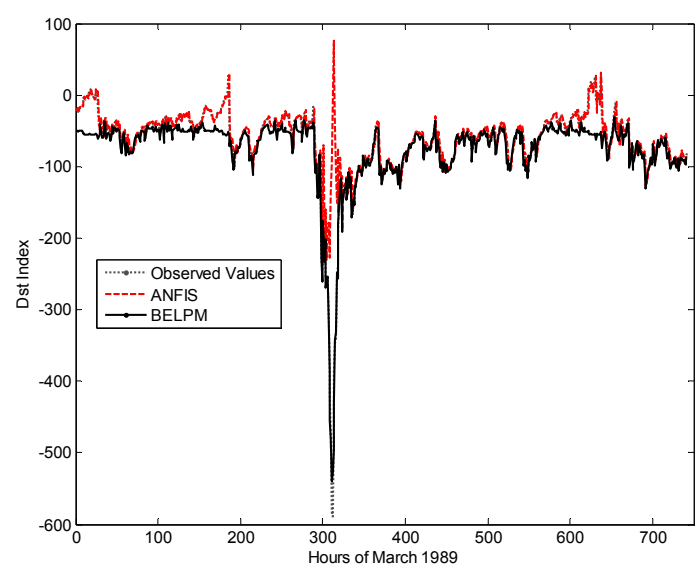

(a)

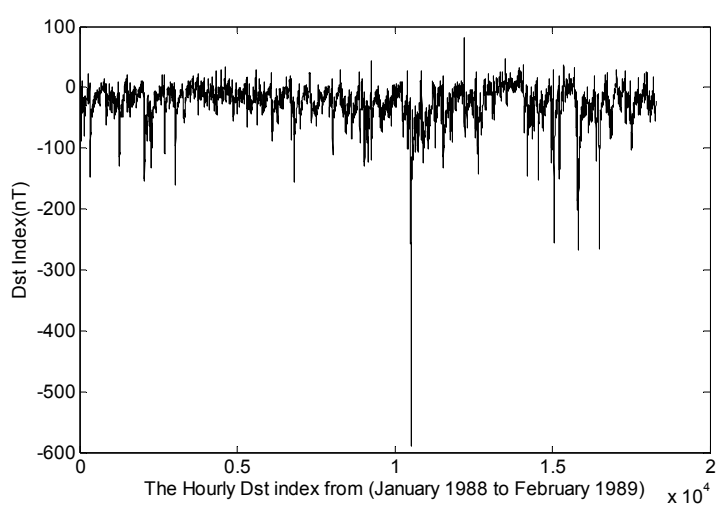

(b)

Fig.11. (a). The observed values and predicted values by ANFIS and BELPM. (b). The training data samples.

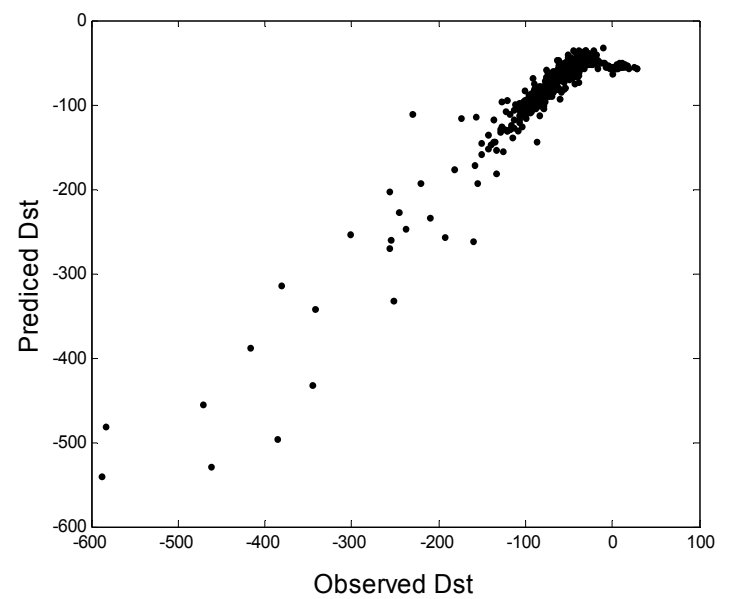

Fig.12. The correlation between the predicted values and the observed values of March 1989.
The correlation between the predicted values and the observed values are shown in Fig. 12. The obtained NMSE of BELPM is 0.1041; while the NMSE index of ANFIS is more than 0.15 .

For the second data set, BELPM is tested for multi-step ahead prediction of the Dst values. The main goal of this case is to evaluate the performance of BELPM for long term prediction of the Dst index. Figure 13. shows the predicted values by BELPM versus the observed values. For one step ahead prediction of the Dst index from $10^{\text {th }}$ to $22^{\text {nd }}$ of July 2000, the NMSE index of BELPM equals to 0.0593; while the NMSE index of ANFIS is equal 0.112. As this figure describes BELPM could predict the Dst index better than ANFIS. Figure 14 shows how increasing the prediction horizon causes increases in the prediction errors of BELPM and ANFIS. It is notable that the values of the NMSE index of BELPM are lower than the values of the NMSE index of ANFIS.

Tabel I compares the performance of three methods on six -steps ahead prediction of the Dst index. It is notable that the BELPM outperforms ANFIS and a neural network method in terms of NMSE and correlation coefficent.

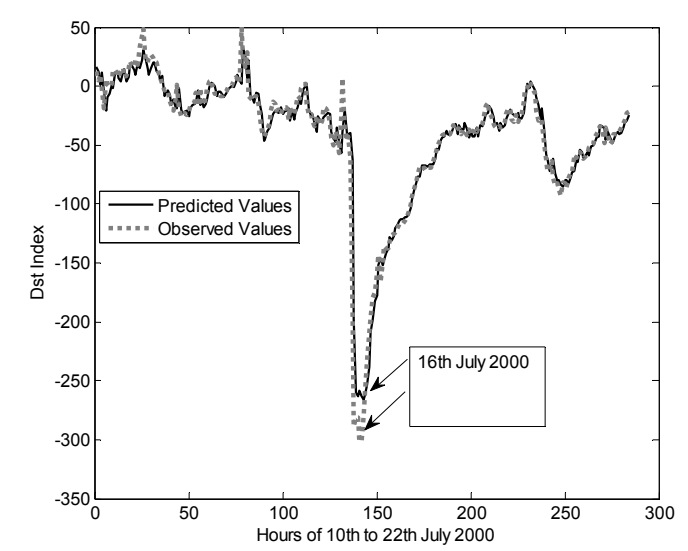

Fig.13.The predicted values of Dst index of 2000

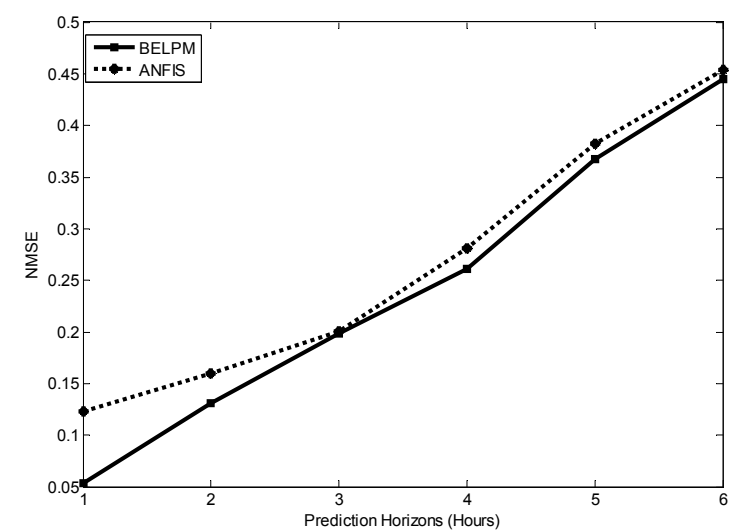

Fig.14. The values of NMSE index versus the prediction horizon of the Dst time series. 
TABLE I

A COMPARISON BETWEEN BELPM, ANFIS AND ANN

Method NMSE $\quad$ Correlation

\begin{tabular}{ccc}
\hline ANN[13] & ---- & 0.75 \\
BELPM & 0.44 & 0.75 \\
ANFIS & 0.45 & 0.74
\end{tabular}

\section{CONCLUSION}

This study has suggested another model of BELIMs that is referred to as Brain Emotional Learning-based Prediction Model (BELPM). This paper examined this model (BELPM) to model the Dst index and has been tested for prediction geomagnetic storms using two data sets of the Dst index. The results have verified that the proposed model can be used for long-term prediction of geomagnetic storms.

As future work, the authors consider adding an evolutionary-based optimization method e.g., genetic algorithm to find optimal values of the fiddle parameters, e.g., the number of neighbors and the initial values of nonlinear parameters. Other improvements in the model would be made on the basis of kd-Tree data structure to address "the curse of dimensionality" problem and decrease the computational time complexity of BELPM. To adjust the nonlinear parameters, different types of optimization methods (e.g., Quasi-Newton or Conjugate Directions) may be utilized. The good results obtained when employing the BELPM for predicting geomagnetic storms are a motivation for applying this model to classify patterns as well as to identify complex systems.

\section{ACKNOWLEDGEMENT}

The authors are grateful for accessing the Dst index provided by NOAA and World Data Center for Geomagnetism and Space Magnetism, Kyoto University

\section{REFERENCES}

[1] L. Xinlin Li, B.Luo and M.Temerin, "Prediction of the Dst, AL, AU Indices Using Solar Wind Parameters,' Geophysical Research Abstracts, Vol. 15, EGU2013-3645, 2013. doi:10.1029/2006JA011918

[2] J. Sharifi, B.N. Araabi and C. Lucas, Multi_step prediction of Dst index using singular spectrum analysis and locally linear neurofuzzy modeling, Earth Planets Space, 2006, vol. 58, pp. 331-341. doi 10.1186/BF03351929.

[3] M. Mirmomeni, and C. Lucas, Analyzing the variation of embedding dimension of solar and geomagnetic activity indices during geomagnetic storm time, Earth Planets Space, 61, 237-247, 2009., doi:10.1186/BF03352904.

[4] M. Mirmomeni, M. Shafiee, C. Lucas, and B. N. Araabi, Introducing a new learning method for fuzzy descriptor systems with the aid of spectral analysis to forecast solar activity, J. Atmos. Sol.-Terr. Phys., 68,2061-2074, 2006. DOI:10.1016/j.jastp.2006.07.001.

[5] http://en.wikipedia.org/wiki/March_1989_geomagnetic_storm

[6] S. Kugblenu, S. Taguchi, and T. Okuzawa, Prediction of the geomagnetic storm associated Dst index using an artificial NN algorithm, Earth PlanetSci., 51, 307-313, 1999.

[7] D. Jankovičová, P. Dolinský, F. Valach, and Z. Vörös, Neural network-based nonlinear prediction of magnetic storms, J. Atmos. Sol. Terr. Phys., 64, 651-656,2002. DOI:10.1016/s1364-6826(02)00025-1
[8] R. Bala, and P. Reiff, Improvements in short-term forecasting of geomagnetic activity, Space Weather, 10, S06001,2012, doi:10.1029/2012SW000779.

[9] H. Gleisner, H. Lundstedt, and P. Wintoft, Predicting geomagnetic storms from solar wind data using time delay neural networks, Ann. Geophys., 14, 679, 1996.

[10] Z. Voros, D. Jankovicova, "Neural network prediction of geomagnetic activity: a method using local Holder exponents," Nonlinear Processes in Geophysics, no. 9, pp. 425 - 433, 2002.

[11] M. Mirmomeni and C. Lucas, "Analyzing the variation of lyapunov exponents of solar and geomagnetic activity indices during coronal mass ejections," Space Weather, vol. 7, p. S07002, July 2009. DOI: 10.1029/2008SW000454

[12] W.K. Tobiska, D. Knipp, W. J. Burke, D. Bouwer, J. Bailey, D. Odstrcil, M. P. Hagan, J. Gannon, and B. R. Bowman (2013), The Anemomilos prediction methodology for Dst, Space Weather, 11, 490508, doi:10.1002/swe.20094.

[13] S. Haykin, Neural Networks: A Comperhensive Foundation.Upper Saddle River, NJ:Prentice Hall, $2^{\text {nd }}$ ed., 1999.

[14] O. Nelles, Nonlinear System Identification:From classicical Approches to Neural Networks and Fuzzy Models. Berlin, Germany: Springer-Verlag, 2001.

[15] T. Babaie, R. Karimizandi, C. Lucas, "Learning based brain emotional intelligence as a new aspect for development of an alarm system,' $J$. Soft Computing., vol. 9, issue 9, pp.857-873, 2008. DOI: $10.1007 / \mathrm{s} 00500-007-0258-8$

[16] A. Golipour,C.Lucas, D. Shamirzadi, Purposeful prediction Of Space Weather Phenomena by Simulated Emotional Learning. Modeling Journal, 24,2004. 65-72.

[17] E. Lotfi and M.R. Akbarzadeh-Totonchi, "Adaptive brain emotional decayed learning for online prediction of geomagnetic activity indices", ;presented at Neurocomputing, 2014, pp.188-196.DOI: 10.1016/j.neucom.2013.02.040

[18] M. Parsapoor, M, U. Bilstrup, "Neuro-fuzzy models, BELRFS and LoLiMoT, for prediction of chaotic time series," in Proc. IEEE Int. Conf. INISTA., pp.1-5, 2012.doi: 10.1109/INISTA.2012.6247025

[19] M. Parsapoor, U. Bilstrup, "Brain Emotional Learning Based Fuzzy Inference System (BELFIS) for Solar Activity Forecasting," in Proc. IEEE Int. Conf. ICTAI 2012, 2012. DOI:10.1109/ICTAI.2012.78

[20] M. Parsapoor, U. Bilstrup, "Brain Emotional Learning Based Fuzzy Inference System (Modified using Radial Basis Function)," $8^{\text {th }}$ IEEE International Joint Conference for Digital InformationManagement, 2013. DOI: 10.1109/ICDIM.2013.6693994.

[21] M. Parsapoor, C. Lucas and S. Setayeshi, "Reinforcement _recurrent fuzzy rule based system based on brain emotional learning structure to predict the complexity dynamic system," in Proc. IEEE Int. Conf. ICDIM, pp.25-32, 2008. Doi: 10.1109/ICDIM.2008.4746712

[22] M. Pasrapoor, U. Bilstrup, "An emotional learning-inspired ensemble classifier (ELiEC)," Computer Science and Information Systems (FedCSIS), 2013 Federated Conference on, vol., no., pp.137,141, 8-11 Sept. 2013.

[23] M. Parsapoor and U. Bilstrup, "Chaotic Time Series Prediction Using Brain Emotional Learning Based Recurrent Fuzzy System (BELRFS)," in International Journal of Reasoning-based Intelligent Systems, 2013. DOI: 10.1504/IJRIS.2013.057273.

[24] M. Parsapoor, Prediction the price of Virtual Supply Chain Management with using emotional methods. M.S. thesis, Dept. Computer. Eng., Science and research Branch, IAU.,

[25] M. Parsapoor, Brain Emotional Learning-Inspired Models. Licentiate dissertation, Halmstad: Halmstad University Press, 2014.

[26] J.Moren, C.Balkenius, "A computational model of emotional learning in the amygdala,' 'in From Animals to Animats, MIT, Cambridge, 2000 .

[27] M. S. Gazzaniga, R. B, Ivry, G.R.Mangun, and Megan.S.Steven, Gognative Nerosc in The Biology of the Mind. W.W.Norton\&Company, New York, $3^{\text {rd }}$ ed., 2009.

[28] J.E.Ledoux, The emotional brain: the mysterious underpinnings of emotional life, Simon \& Schuster,NY ,1998.

[29] C. Lucas, D. Shahmirzadi, N. Sheikholeslami, "Introducing BELBIC: brain emotional learning based intelligent controller,' J. INTELL. AUTOM. SOFT. COMPUT., vol. 10, no. 1, pp. 11-22, 2004. DOI: $10.1080 / 10798587.2004$ 
[30] N. Sheikholeslami, D. Shahmirzadi, E. Semsar, C. Lucas., "Applying Brain Emotional Learning Algorithm for Multivariable Control of HVAC Systems,", J. Intell. Fuzzy. Syst. Vol. 16, pp. 1-12, 2005.

[31] A. R. Mehrabian, C. Lucas, J. Roshanian,"Aerospace Launch Vehicle Control: An Intelligent Adaptive Approach", J. Aerosp. Sci. Technol., vol. 10, pp. 149-155, 2006. DOI: 10.1016/j.ast.2005.11.002
[32] R. M. Milasi, C. Lucas, B. N. Araabi, "Intelligent Modeling and Control of Washing Machines Using LLNF Modeling and Modified BELBIC," in Proc. Int. Conf. Control and Automation., pp.812-817, 2005. DOI: 10.1109/ICCA.2005.1528234

[33] A. M. Yazdani1, S. Buyamin1, S. Mahmoudzadeh2, Z. Ibrahim1 and M. F. Rahmat1., "Brain emotional learning based intelligent controller for stepper motor trajectory tracking," J. IJPS., vol. 7, no. 15, pp. 2364-2386, 2012. DOI: 10.5897/IJPS11.1590 